\title{
Health-related quality of life, lung function and dyspnea rating in COPD patients
}

\author{
M. Justine, F. Tahirah, V. Mohan
}

ABSTRACT: Health-related quality of life, lung function and dyspnea rating in COPD patients. M. Justine, F. Tahirah, V. Mohan.

Background and Aim. COPD is a progressive and irreversible disease, thus assessing the impact of the disease on health-related quality of life (HRQOL) is important in the management of COPD. The aim of this study was to examine the relationship between HRQOL, lung function and dyspnea rating in patients with stable COPD.

Methods. One hundred COPD patients (mean age = 64.76 \pm 11.43 years) were recruited for this cross-sectional study. Lung function test was measured using a FlowScreen portable spirometry. Dyspnea rating was measured using the baseline dyspnea index (BDI). HRQOL was assessed using the SF-36v2 which summarized two components; physical health component summary (PHCS) and mental health component summary (MHCS).
The results. The mean value of lung function (Forced expiratory volume in 1 second, $\mathrm{FEV}_{1} \%$ predicted) was $58.19 \pm 30.24$ and dyspnea rating was $6.85 \pm 2.68$. The lung function was significantly correlated with MHCS $(r=.294$, $p<0.05)$ but not with the PHCS $(p>0.05)$. The dyspnea rating was significantly correlated with both PHCS $(r=.730$, $\mathrm{p}<0.05)$ and MHCS $(\mathrm{r}=.324, \mathrm{p}<0.05)$. Regression analysis indicated that dyspnea rating emerged as the most significant predictor for PHCS and MHCS accounting for 54\% and $12 \%$ of the variances respectively.

Conclusions. The findings show that dyspnea rating is an important factor in predicting HRQOL of patients with COPD. This indicates that dyspnea rating influences HRQOL to a greater extent than the physiological measurement of lung function. Therefore, focusing on such predictors at an early stage may provide meaningful benefits in the management of COPD.

Monaldi Arch Chest Dis 2013; 79: 3-4, 116-120.

Keywords: COPD, Dyspnea, Lung function, Quality of life.

Physiotherapy Department, Faculty of Health Sciences, Universiti Teknologi MARA, Puncak Alam Campus, Selangor Malaysia.

Correspondence: Maria Justine, Physiotherapy Department, Faculty of Health Sciences, Universiti Teknologi MARA, Puncak Alam Campus, 42300 Puncak Alam, SelangorDarulEhsan, Malaysia; e-mail:mar_physio@yahoo.com

\section{Introduction}

COPD is one of the principal causes of major morbidity and mortality that affects the population worldwide with higher incidence in men than women [1]. Age and smoking were found to be the strongest contributors to COPD [2,3]. Age is considered a non-modifiable factor that accompany the progression of the disease. In contrast, a study conducted in Abu Dhabi among 40-80 year-old subjects, associations between COPD with cigarette smoking or with other local inhaled exposures were not observed [4]. This could be related to the low prevalence of smoking among this population (12\% current and $12 \%$ former smokers) [4].

Asia has been found to be one of the regions with high prevalence of COPD. A study conducted among populations aged 30 years and older revealed that the number of moderate to severe COPD cases in the 12 countries of this region is 56.6 million with an overall prevalence rate of 6.3\% [5]. In Spain, diagnosed patients with COPD normally had severe disease and more severely impaired health-related quality of life (HRQOL) while patients with undiagnosed COPD stage 1+ also showed impairment in HRQOL and in some aspect of ADL [3]. Various outcome measures had been used to measure the quality of life of COPD patients and mostly showed consistent findings in the reduction of HRQOL [6-9].

At present, there are no epidemiological sound longitudinal follow-up of populations that can provide an accurate picture of the success or failure, of the efforts that over the past few decades have been made to prevent and treat COPD [10]. The current methods for assessing the clinical outcomes in COPD mainly rely on physiological tests $\left(\mathrm{FEV}_{1}\right.$ measurements) combined with the use of questionnaires [11]. While it is well understood that COPD is a progressive and irreversible disease, thus assessing the impact of the disease on HRQOL is rather an important aim in the management of COPD. Furthermore, continuous efforts on identifying the optimal methods for assessing the outcomes of COPD on HRQOL may provide a meaningful clinical outcome especially in maintaining the daily function of patients with COPD despite the presence of a breathing difficulty.

Therefore, the purpose of this study was to examine the relationships between HRQOL (physical health and mental health component summaries) with lung function and dyspnea rating. This study also sought to answer whether lung function and/or dyspnea rating significantly pre- 
dict the HRQOL. The findings of this study may be significant for healthcare providers in deciding which factor could be the target for COPD management.

\section{Materials and Methods}

\section{Sample}

A group of 100 stable COPD patients, who attended outpatient medical treatment, was recruited for this cross-sectional correlational study. The criteria for subject inclusion in the study were as follows: a diagnosis of COPD; $\mathrm{FEV}_{1} / \mathrm{FVC}$ less than 70 percent, age 40 years and above, Malaysian citizens, and has less than 5 co-morbidities. Criteria for exclusion were as follows: disabled (wheelchair dependent), diagnosed with cancer, uncontrolled diabetes, uncontrolled hypertension, psychiatric illness, exertional chest pain, musculoskeletal or neurologic disease with functional limitation, liver or renal failure, unstable gastrointestinal condition, current substance abuse problem, and legally blind or deaf. Each subject was required to sign a written consent form. The protocol for this study was approved by the ethics committee of the local university.

\section{Data Collection}

HRQOL, lung function and dyspnea rating were assessed in each patient on the same day of their visit to the outpatient clinic. The sequence of data collection was selected so that scores of dyspnea rating and HRQOL would be obtained prior to the results of the physiological tests. Subjects' demographic and health characteristics which include gender, age, duration of illness, body weight, height, body mass index, comorbidities, smoking history and type of medication taken were recorded.

The HRQOL was measured using the SF$36 \mathrm{v} 2$, a questionnaire that covers two summary measures; physical health component summary (PHCS) and mental health component summary (MHCS). The questionnaire is easy to use, and has been validated in several languages, including Malay [11]. The questionnaire was found to be a valid instrument to measure the impact of COPD on HRQOL [12], and shown to be discriminative as well as responsive to long-term disease progression [11]. The total score for each component ranges from 0 to 100 . The higher the score the better the HRQOL is.

Lung function was measured in a seated position using a portable Spirometry (Model: Flowscreen: VIASYS, SN 38201743, 240Hz, $50 / 60 \mathrm{~Hz}, 60 \mathrm{Watt})$. The system sets met the criteria for standardization and were calibrated prior to testing. $\mathrm{FEV}_{1}$ predicted normal values were taken from the asian population percentage by calculating the height and weight of the subject [13]. A minimum of three efforts was performed until a reproducible maximal value were obtained.

Dyspnea rating was measured using the baseline dyspnea index (BDI), a multidimensional in- strument for measuring breathlessness based on three components that may evoke dyspnea: functional impairment, magnitude of the task and magnitude of the effort. A previous study has demonstrated a good validity and reliability of the BDI in patients with COPD [14]. Patients may select from one of five grades of dyspnea based on a brief history of each of the three components of the BDI. This process took about 5 minutes to complete. The grading of the indexes was such that the lower the score reflecting the more severe the breathlessness perceived [14].

\section{Statistical Analysis}

Descriptive analysis was conducted to present the means, standard deviations, and ranges for each variable of interests. Examination of the frequencies and analysis of the data indicated that all variables were normally distributed without any outliers.

Correlational analyses using the Pearson's correlation coefficient ( $\mathrm{r}$ ) were conducted to determine the degree of association between i) physical health component summary (PHCS) with lung function and dyspnea rating; and ii) mental health component summary (MHCS) with lung function and dyspnea rating. A p-value of less than 0.05 was considered significant. A regression analysis was conducted to further investigate whether variables that were significantly correlated reliably predict the score for PHCS and MHCS. Examination on the bivariates correlation matrices, variance inflation factor (VIF) values, and tolerance criteria indicated no multicollineriaty among the independent variables.

\section{Results}

The mean age of the participants for this study was $63.76 \pm 11.43 \mathrm{yr}( \pm \mathrm{SD})$ (range, $40-84$ years), $84 \%$ of the respondents were male and $16 \%$ were female, mean height was $166.66 \pm 7.51 \mathrm{~cm}$ (range, $142-179 \mathrm{~cm})$, and mean weight was $60.87 \pm 16.24$ $\mathrm{kg}$ (range, 26.2-131.85 kg). With regards to the respondents' health history, $64 \%$ of the subjects were ex-smokers, $22 \%$ were current smokers and $14 \%$ were non-smokers. Most of the respondents presented with 2 to 4 co-morbidities and none of the respondents had more than 5 co-morbidities with a mean of $4.076 \pm 4.396$ years of duration of illness.

Values for the PHCS, MHCS, lung function and dyspnea rating are shown in Table 1.

Table 2 shows the results of the correlational analysis. There were significant positive strong relationships between dyspnea rating with PHCS $(\mathrm{r}=.736, \mathrm{p}<0.05)$, and significant positive moderate relationship with MHCS $(\mathrm{r}=.346, \mathrm{p}<0.05)$. There was a statistically significant low correlation between lung function with PHCS ( $\mathrm{r}=0.267$, $\mathrm{p}<0.05$ ) but no significant correlation with MHCS $(\mathrm{r}=0.095, \mathrm{p}>0.05)$. These results indicate patients who perceived higher breathing capacity (dyspnea rating) were also likely to obtain a higher quality 
Table 1. - Dyspnea rating, health-related QOL and lung function of study participants

\begin{tabular}{lcc}
\hline Variables & Mean \pm SD & Range \\
\hline Dyspnea rating & $6.85 \pm 2.68$ & $0-12$ \\
\hline Health-related quality of life (HRQOL) & & \\
$\quad$ PHCS & $41.69 \pm 7.99$ & $20.21-56.68$ \\
MHCS & $46.47 \pm 13.14$ & $11.74-74.66$ \\
\hline Lung function (FEV $\%$ predicted) & $58.19 \pm 30.24$ & $30.7-100$ \\
\hline
\end{tabular}

explained that patients with COPD frequently decrease their activity in order to avoid the unpleasant sensation of breathlessness which in turn impact their quality of life. Another possible explanation for this may be that dyspnea significantly influences HRQOL as both dyspnea and HRQOL are assessments of a patient's perspectives [19]. Dyspnea pro-

of life. While patients who have higher lung function were also likely to present with higher PHCS but not MHCS.

A stepwise multiple regression was conducted to determine whether dyspnea rating and/or lung function significantly predict PHCS. The overall model was found to be statistically significant $\left(\mathrm{R}_{2}=.543, \mathrm{~F}=58.209, \mathrm{p}=0.001\right)$. However, the dyspnea rating alone explained about $53.7 \%$ of the variance in $\mathrm{PHCS}\left(\mathrm{R}_{2}=.537, \mathrm{~F}=114.83, \mathrm{p}=0.001\right)$.

A bivariate regression was conducted to determine the predictor for mental health. Since the lung function was not significantly correlated with MHCS, then the MHCS was regressed only on the dyspnea rating. The model was statistically significant $\left(\mathrm{R}_{2}=0.115, \mathrm{~F}=12.527\right.$, beta $\left.=1.615, \mathrm{p}<0.05\right)$ which indicated that dyspnea rating explained about $11.5 \%$ of the variance in MHCS.

\section{Discussion}

\section{Dyspnea rating and $\mathrm{HRQOL}$}

There was a statistically significant positive strong relationship between dyspnea rating with PHCS and statistically significant positive moderate relationship with MHCS. Therefore it can be concluded that differences in the clinical rating of dyspnea may influence the quality of life in patients with COPD. This finding is consistent with a previous study which stated that the severity of dyspnea was a significant predictor for HRQOL [12]. Similar conclusions were drawn from various other studies [15-17].

The possible reason for the finding may be due to the problem whereby dyspnea exerts a major effect on COPD patient's ability to perform various daily activities, thus it interferes with the patient's HRQOL. This is supported by Siafakas [18] who

Table 2. - Results of the analyses of Pearson's correlation

\begin{tabular}{lcc}
\hline $\begin{array}{l}\text { Components } \\
\text { of HRQOL }\end{array}$ & $\begin{array}{c}\text { Dyspnea rating } \\
\mathbf{r}\end{array}$ & $\begin{array}{c}\text { Lung function } \\
\mathbf{r}\end{array}$ \\
\hline PHCS & $.736^{*}$ & $.267^{*}$ \\
\hline MHCS & $.344^{*}$ & .095 \\
\hline$*$
\end{tabular}

* significant at $\mathrm{p}<0.05$. vides information about the impact of respiratory impairment on the patient's HRQOL which can be considered to be the most important aspect of COPD from a patient's perspective [20]. While Jones et al [21], who have found a significant correlation between dyspnea rating and PHCS, explained that dyspnea is the predominant symptom of most of the COPD patients which is the main target for medical management. Thus, it may be concluded that by encountering dyspnea, one may improve their quality of life.

\section{Lung function and HRQOL}

There is a statistically significant low correlation between lung function and PHCS, whereas no significant correlation was found between lung function with MHCS. Therefore, it can be concluded that changes in the lung function may or may just slightly affect the health-related quality of life among COPD sufferers.

The results of this research are consistent with a number of previous studies, who reported low correlation between lung function and quality of life [12, 16, 22-24]. In contrast, a few studies reported no significant correlation between lung function and quality of life $[15,25]$.

Lung function, as measured by the $\mathrm{FEV}_{1}$, may not be influenced by the quality of life which can be due to several factors. According to Glaab [11], this could be attributed to the $\mathrm{FEV}_{1}$ measurements that is based on an artificial maneuver that may not really represent the functional adaptation of patients with COPD. It was also highlighted that the change in patient's symptoms may occur against a relatively modest change in lung function, thus the reduction in $\mathrm{FEV}_{1}$ may not correlate well with patient's HRQOL [19, 20]. While Jones et al [21] discussed that there may be improvements in quality of life with only minimal changes in $\mathrm{FEV}_{1}$. This is because although $\mathrm{FEV}_{1}$ measures reflect direct changes in airflow limitation, the measure of the symptom is much more important in revealing the burden of the disease in the patient's everyday life. Thus the $\mathrm{FEV}_{1}$ measure alone does not really reflect the nature and the burden associated with COPD.

Other than that, confounding factors such as age, smoking history and other comorbidities may affect the findings on quality of life without any real changes in lung function and vice versa. For example, at a younger age one may have a better 
quality of life although with poor $\mathrm{FEV}_{1}$. The presence of other comorbidities may also impact on the quality of life without deteriorations in $\mathrm{FEV}_{1}$. While smoking habits may definitely influence $\mathrm{FEV}_{1}$ results, most smokers feel that their days are incomplete without smoking [26]. Thus people who are satisfied with their everyday lives are the ones that perceive a good quality of life.

Another argument is that the selection of the instrument which is a generic measure for HRQOL. According to Chen et al [27], SF-36 include most of the essential concepts of HRQOL and have been shown to be suitable for cross-cultural applications irrespective of their type or number of illness. However, they may be not sensitive to some problems unique to particular disease [27]. Thus, it may be more accurate to use a disease specific measure such as the St George's Respiratory Questionnaire (SGRQ). A recent study has shown that the SGRQ demonstrated greater reliability in discriminating among different levels of severity stages of COPD than generic measures of health, which suggests that the SGRQ may provide COPD studies with greater statistical power than the SF-36 summary in capturing meaningful differences in clinical severity [28].

According to our data, dyspnea level influences the quality of life to a greater extent than physiologic measurements do in patients with COPD. Thus, it is imperative that clinicians treat not only the medical illness itself but also the concomitant physical symptoms in order to optimize the quality of life of people with COPD. A shift of focus from the pathophysiology of disease to assessment and relief of symptoms may provide meaningful benefits for COPD patients in terms of enhancement of quality of life.

For the health care professionals, the selection of measurement tools in clinical practice to quantify symptoms as well as the overall health status are essential. Thus, the implementation of the BDI and SF-36 outcome measures in the assessment of COPD patients' physical function should be put forward. However, the limitation of using the SF36 is that as a generic measure, it is considered less responsive than disease-specific instruments in COPD and is not consistently responsive to therapeutic effects [11]. As suggested by Chen et al [27] a combination of generic and disease specific measure of HRQOL may be more appropriate for monitoring changes in a patient's health status due to an intervention. Thus, it is recommended that future studies involving similar population background may need to be undertaken in order to more accurately measure the quality of life over time along with the progression of the disease using a disease-specific outcome measure such as the SGRQ.

Acknowledgements: The authors are indebted to the staff at Lung Function Laboratory at the Institute of Pulmonary Rehabilitation, Kuala Lumpur and all of the subjects for their assistance and cooperation for the successful completion of the study.

\section{References}

1. Afonso ASM, Verhamme KMC, Sturkenboom MCJM, Brusselles GGO. COPD in the general population: Prevalence, incidence and survival. Respir Med 2011; 105: 1872-84.

2. Buist AS, McBurnie MA, Vollmer WM, et al. International variation in the prevalence of COPD (The BOLD Study): A population-based prevalence study. Lancet 2007; 370: 741-50.

3. Miravitlles M, Soriano JB, Garcia-Rio F, et al. Prevalence of COPD in Spain: Impact of undiagnosed COPD on quality of life and daily life activities. Thorax 2009; 64: 863-8.

4. Al Zaabi A, Asad F, Abdou J, et al. Prevalence of COPD in Abu Dhabi, United Arab Emirates. Respir Med 2011; 105: 566-70.

5. Tan WC, Seale JP, Charaoenratanakul S, et al. COPD prevalence in 12 Asia- Pacific countries and regions: Projections based on the COPD prevalence estimation model. Respirology 2003; 8: 192-8.

6. Berry CE, Drummond MB, Han MLK, et al. Relationship between lung function impairment and health-related quality of life in COPD and interstitial lung disease. Chest 2012; 142: 704-11.

7. Han MLK, Curran-Everett D, Dransfield MT, et al. Racial differences in quality of life in patients with COPD. Chest 2011; 140:1169-76

8. Sundh J, Ställberg B, Lisspers K, Montgomery SM, Janson C. Co-morbidity, body mass index and quality of life in COPD using the clinical COPD Qqestionnaire. Chest 2011; 8: 173-81.

9. $\mathrm{Xu} \mathrm{W}$, Collet JP, Shapiro S, et al. Negative impacts of unreported COPD exacerbations on health-related quality of life at 1 year. Eur Respir $J$ 2010; 35:1022-30.

10. Celli BR. The light at the end of the tunnel: is COPD prevalence changing. Eur Respir J 2010; 36: 718-19.

11. Glaab T, Vogelmeier C, Buhl R. Outcome measures in chronic obstructive pulmonary (COPD); strength and limitations. Respir Res 2010; 11: 79.

12. Mahler DA, Mackowiak JI. Evaluation of the ShortForm 36-Item questionnaire to measure health-related quality of life in patients with COPD. Chest 1995; 107: 1585-9.

13. Singh R, Singh HJ, Sirisinghe RG. Sprometric studies in Malaysians between 13 and 69 years of age. Med $J$ Malaysia 1993; 48: 175-184.

14. Mahler DA, Weinberg DH, Wells CK, Feinstein AR. The measurement of dyspnea: contents, interobserver agreement, and physiologic correlates of two new clinical indexes. Chest 1984; 85: 751-758.

15. Hu J, Meek P. Health-related quality of life in individuals with chronic obstructive pulmonary disease. Heart Lung 2005; 34: 415-22.

16. Mahler DA, Faryniarz K, Tomlinson D, et al. Impact of dyspnea and physiologic function on general health status in patients with chronic obstructive pulmonary disease. Chest 1992; 102: 395-401.

17. Viramontes JL, O'Brien B. Relationship between symptoms and health related quality of life in chronic lung disease. J Gen Intern Med 1994; 9: 46-8.

18. Siafakas NM, Anthonisen NR, Georgopoulos D. Acute exacerbations of chronic obstructive pulmonary disease. Marcel Dekker Inc. New York, 2004.

19. Linus H, Tomas S, Varkey B. Improving health-related quality of life in chronic obsructive pulmonary disease. Curr Opin Pulm Med 2004; 10: 120-7.

20. Ries AL. Impact of chronic obstructive pulmonary disease on quality of life: The role of dyspnea. Am J Med 2006; 119: S12-S20.

21. Jones P, Lareau S, Mahler DA. Measuring the effects of COPD on the patient. Respir Med 2005; 99: S1-S18. 
22. Hajiro T, Nishimura K, Tsukino M, Ikeda A, Koyama $\mathrm{H}$, Izumi T. Analysis of clinical methods used to evaluate dyspnea in patients with chronic obstructive pulmonary disease. Am J Respir Crit Care Med 1998; 158: 1185-9.

23. Stahl E, Lindberg A, Jansson S, et al. Health-related quality of life is related to COPD disease severity. Health Qual Life Outcomes 2005; 3: 56.

24. Terence AR, Gavin C, Elizabeth A, et al. Effect of exacerbation on quality of life in patients with chronic obstructive pulmonary disease. Am J Respir Crit Care Med 1998; 157: 1418-22.

25. Seung Kim HS, Kunik ME, Molinari VA, et al. Func- tional impairments in COPD patients: The impact of anxiety and depression. Psychosomatics 2000; 41: 6.

26. Spiter J. You Smoke Because You're a smoke-a-holic! Joel's Reinforcement Library, 2000. Retrieved April 22, 2009 from www.yahoo.com

27. Chen TH, Li L, Kochen MM. A systematic review: How to choose appropriate health-related quality of life (HRQOL) measures in routine general practice? $J$ Zhejiang Univ SCI 2005; 6B (9): 936-940.

28. Pickard AS, Yang Y, Lee TA. Comparison of healthrelated quality of life measures in chronic obstructive pulmonary disease. Health Qual Life Outcomes 2011; 9: 26.

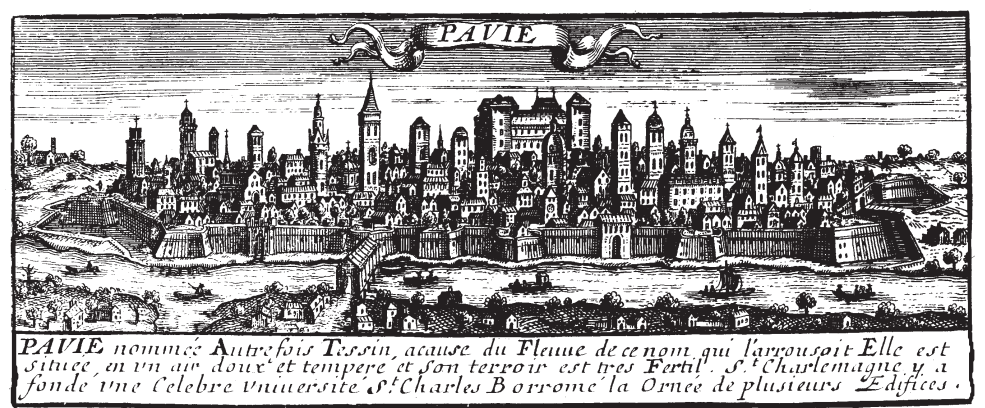

